\title{
LA NOTION DE MODÈLE DE DISCOURS APPLIQUÉE À LA TRADUCTION
}

\begin{abstract}
A bstract. Bocquet Claude, La notion de modèle de discours appliquée à la traduction [The concept of the model of discourse as applied in translation]. Studia Romanica Posnaniensia. Adam Mickiewicz University Press, Poznań, vol. XXV/XXVI: 2000, pp. 21-27. ISBN 83-232-0965-0. ISSN 0137-2475.
\end{abstract}

The author makes an attempt at determining how much and to what an extent various types of discourse and their characteristics have, or should have, an influence on the process of translation. In the text it is shown in what way the medical discourse historically had an influence on the modern French in the field of economics. In order to draw conclusions concerning translation of this type of texts this kind of special French is compared with the German and Italian language of economics.

\section{LES DIVERS TYPES DE DISCOURS SPÉCIALISÉS ET LA TRADUCTION}

\subsection{PRÉSAVOIR ET LANGAGE}

Voltaire avait des idées bien tranchées sur la traduction, qui le rattachaient aux belles infidèles en vogue dans le salon d'Houdard de la Motte, qu'il fréquentait. Mais qu'elle ne fut pas sa surprise lorsque, voulant traduire un traité d'astronomie de Newton, il s'aperçut qu'il ne lui suffisait pas pour réussir l'opération de savoir parfaitement l'anglais et le français mais qu'il était tout aussi indispensable de comprendre l'astronomie. Il venait de prendre conscience du problème du présavoir, connu bien avant lui d'ailleurs, puisqu'il est présenté par Etienne Dolet dans La Manière de bien traduire d'une langue en aultre en 1540. Voltaire dut donc avoir recours à son ami de l'époque, Moreau de Maupertuis, mathématicien mais également un peu astronome et spécialiste des langues, pour mener à bien son travail de traduction. La question du présavoir nécessaire à la traduction est devenue un des classiques de la traductologie. On peut dire qu'elle a un caractère non linguistique. Toute différente est 
la question des types de discours auxquels le traducteur doit faire face. Nous emploierons ici indistinctement les concepts de langage et de discours, non que nous leur attribuions le même sens, mais le premier est l'instrument du second.

Qu'est-ce qu'un type de discours? Il se trouve que les praticiens de chaque science ont pris des habitudes langagières qui distinguent la forme linguistique de leur discours de celle des autres discours. Cela tient à une multitude de facteurs, comme l'attachement de certaines pratiques à une forme désuète de langage, à un codage d'initiés, ou à une forme elliptique destinée à accélérer le transfert du message: cela pour ne prendre que quelques exemples. Ces pratiques ne font pas pour autant de ces langages de spécialités de vraies langues autonomes, mais des formes de discours originales; cela se situe plus au niveau de la parole que de la langue. Cela étant, il se trouve que les langages de spécialités varient d'une langue à l'autre selon les critères les plus divers. Et là le traducteur se trouve en difficulté. Il doit entrer dans la problématique linguistique des spécialistes de la matière envisagée dans chacune des deux langues. Les traductologues de notre siècle ont souvent étudié la question. C'est ici que se trouve la première source de la réflexion d'Edmond Cary lorsqu'il définit sa fameuse typologie des textes à traduire dans Comment faut-il traduire et qu'il conclut que «chaque genre a ses règles propres». Plus près de nous dans le temps, et dans la sphère germanique, Katharina Reiß a construit toute sa théorie sur la même exigence d'une typologie.

\section{TRADUCTION SPÉCIALISÉE}

\subsection{LANGAGE TECHNIQUE ET TERMINOLOGIE}

Les théories les plus élémentaires de la traduction aiment à distinguer la traduction générale et la traduction qu'elles appellent technique. Cette dernière est à leurs yeux caractérisée par une suprématie de la terminologie comparée, chaque terme scientifique de la langue source devant correspondre à un et un seul terme de la langue cible. Pour le reste, le discours des sciences se traduirait d'une façon mécanique. Une telle conception des choses, dénoncée avec ironie par Jean-René Ladmiral, se fonde sur un a priori indiscuté, sur un postulat, si l'on veut, celui selon lequel les diverses cultures attachées aux diverses langues n'auraient aucun impact sur la manière de concevoir la matière scientifique. A vrai dire, c'est aussi le postulat qui fonde l'opinion de ceux qui veulent faire de l'anglais la langue universelle, et finalement unique, de toutes les sciences. Dans une des dernières livraisons de la revue mensuelle du rectorat de l'Université de Genève, un professeur de sciences politiques, c'est-à-dire un spécialiste d'une science qui ne peut se cacher derrière l'universalité des graphes et des opérations mathématiques, traitait de simples réactionnaires tous ceux qui n'admettent pas cette universalité de l'anglais. Il semblait simplement ne pas se rendre 
compte qu'en exigeant cette suprématie d'une seule langue, qu'elle soit l'anglais, le latin, l'espéranto ou le volapük, il affirmait implicitement que toutes les sciences sont universelles au point de ne rien devoir aux langues dans lesquelles les penseurs les conçoivent et les expriment, que les langues ne sont que de purs moyens de transmission tout à fait neutres, aussi neutres que la feuille de papier quadrillée ou lignée qui porte le message d'une lettre et que finalement il est inadmissible de croire qu'un chimiste, qu'un politologue ou qu'un médecin puisse voir sa science différemment selon la culture à laquelle il appartient. Les traducteurs, les interprètes et les linguistes sont parmi les mieux à mềme de se rendre compte que cette position est erronée.

\subsection{LES LANGUES DE SPÉCIALITÉ À SOURCE AUTONOME: LE CAS DU LANGAGE MÉDICAL}

Il est des sciences très anciennes, celles qui remontent souvent à l'Antiquité et qu'ont enseignées les universités dès leur origine au Moyen Age, sous une forme ou sous une autre et sous des appellations diverses: pensons aux mathématiques, à la logique, à la physique, à la chimie, à la médecine. Pour ces sciences, un langage s'est constitué dans chaque langue au travers des siècles à mesure même que les langues modernes se constituaient elles-mềmes. Les sources en sont les langues classiques, surtout le grec, le latin et l'arabe. Disons que, pour ces sciences-là, le langage est autonome en cela qu'il n'a pas pour référence le lángage d'autres disciplines. A cet égard, le langage médical des différentes langues modernes est tout à fait symptomatique: selon les langues envisagées, tant la terminologie que le jeu métaphorique que l'ensemble du discours empruntera plus ou moins à l'arabe ou au grec, mais finalement ce discours médical n'aura jamais de référence dans le discours d'autres sciences. C'est la raison pour laquelle le traducteur pourra souvent croire, avec prudence, à des équivalences biunivoques d'une langue à l'autre.

\subsection{LE CAS DU DISCOURS JURIDIQUE}

Parmi les sciences universitaires anciennes, il faut bien sûr citer la science juridique. Quant à ses sources, le discours juridique ne fait pas vraiment exception par rapport aux autres sciences anciennes. Le caractère exceptionnel de la traduction juridique, qui exclut le terminologisme et les correspondances biunivoques, tient à un tout autre fait: l'absence d'homogénéité de l'objet sur lequel porte le discours, du fait des différences institutionnelles. C'est sans doute de là que vient la position, erronée à notre sens, d'Emmanuel Didier qui, ne pouvant traiter la traduction juridique comme la traduction spécialisée appliquée aux autres sciences anciennes, en arrive à rétrécir le sens même du concept de traduction juridique aux seuls rares cas où elle peut être 
traitée comme la traduction appliquée aux autres sciences: ceux où il y a unité institutionnelle, ce qu'on pourrait appeler en termes linguistiques unité de référent. La question reste marginale par rapport à notre sujet.

\section{DES TYPES DE DISCOURS AUX MODÈLES DE DISCOURS}

\subsection{LES CONDITIONS D'EXISTENCE DES MODĖLES}

Pour les sciences anciennes, un type de discours s'est donc constitué en même temps que les langues modernes elles-mêmes. C'est là une situation relativement confortable pour le traducteur. Restent toutes les sciences qui sont apparues au XVIII ${ }^{\mathrm{e}}$ et au XIX ${ }^{e}$ siècles, voire au $\mathrm{XX}^{\mathrm{e}}$ siècle: l'économie, la sociologie, la science politique, voire... la traductologie. Elles sont nées alors que les langues modernes étaient déjà solidement établies: les modèles anciens leur étaient ainsi fermés. Et par ailleurs, ces sciences nouvelles ont eu des origines souvent fort différentes dans chaque culture. $\mathrm{N}$ 'ayant pas la possibilité de constituer un discours autonome, elles se sont donc constitué un discours par référence à celui d'une ou de plusieurs sciences anciennes. C'est cela que nous appelons les modèles de discours. Or il se trouve que les aléas de l'histoire qui ont fait naître les sciences nouvelles, les ont rattachées à des modèles qui sont de plus variables d'une culture à l'autre.

\subsection{L'EXEMPLE DU DISCOURS ÉCONOMIQUE}

Le thème que nous venons de soulever ainsi n'a été que relativement peu étudié sous cet angle. Il peut entraîner de très vastes recherches pour chacun des discours envisagés dans chacune des langues envisagées. Nous nous contenterons ici d'un de ces discours que nous connaissons le mieux: le discours économique, dans la perspective des trois langues et cultures que nous connaissons le mieux: le français, l'allemand et l'italien. La science économique est née en Grande-Bretagne et en France au XVIII ${ }^{\mathrm{e}}$ siècle. En France elle a eu pour premier représentant des dilettantes, dont les deux plus illustres sans doute furent des médecins: François Quesnay, le maître de l'Ecole des Physiocrates, et Clément Juglar, dont on se rappelle l'analyse de la théorie des cycles économiques. Ces gens-là maîtrisaient parfaitement le discours d'une science ancienne: la médecine. Ils pouvaient donc prendre comme modèle de discours de leur science nouvelle: l'économie, celui de leur science ancienne: la médecine. Au-delà du modèle de discours, c'est aussi un modèle de pensée et d'analyse qui allait s'imposer à eux: l'économie allait ainsi avoir une anatomie, une physiologie, une pathologie et des modes de traitement. Le langage et le discours économiques français était né. Deux siècles plus tard, il ne s'est guère éloigné de son modèle 
initial. Preuve en soit le discours que nous rencontrons aujourd'hui encore à toutes les pages des revues économiques. Quelques exemples au hasard tirés de la presse économique:

Cette accumulation de paralysie ici et de freinage là explique la langueur de l'économie mondiale. (...)

Les Américains ont cherché à redonner du tonus à leur économie. Les marchés du travail se dégradent plus vite qu'ils n'entrent en convalescence.

(Numéro spécial de Dossiers et Documents du Monde, janvier 1992: Bilan économique et social).

Pays sous perfusion, le Tchad vit de l'aide publique internationale. (...)

L'étrange maladie des caisses d'épargne américaines a pris des allures d'épidémie cette année rendant nécessaire un traitement de choc. (...)

La convalescence des grandes banques américaines est bel et bien terminée. La baisse des taux leur a permis de se refaire une santé. Deux des plus grandes banques du pays ont été mises sous perfusion par l'Etat. Sur un marché japonais en pleine déprime depuis que l'économie ne croît plus à un rythme inférieur à $2 \%$.

(Le Monde diplomatique, février 1993).

C'est que les gains de rentabilité de nos places de travail ont été confisqués par l'injection parfaite d'une main-d'oeuvre dont les emplois s'avèrent maintenant superflus. (..)

L'anatomie de l'économie réelle de notre pays nous échappe au plan du fonctionnement de ses organes et de ses cellules.

(L'Hebdo $\mathrm{N}^{\circ} 47 / 92$ ).

Et de formules inquiétantes comme:

Le prix du lait amputé dans la douleur.

(L'Hebdo $N^{\circ} 47 / 92$ ).

Et plus inquiétant encore:

A quand un Paracelse de l'analyse économique suisse pour autopsier la dépouille de la dernière haute conjoncture, un docteur ès économies qui nous indique la cure pour les années à venir.

(L'Hebdo N47 / 92).

On voit ici qu'on pratique l'autopsie avant de penser à une cure, mais peu importe.

En Allemagne, l'économie est née dans le prolongement de la science du droit, parce qu'elle a été pratiquée d'abord par des juristes, les juristes de l'Ecole historique. Leur modèle de discours a donc été le discours juridique. Les économistes allemands de la fin du $\mathrm{XX}^{\mathrm{e}}$ siècle raisonnent encore en terme de loi, loi naturelle, de dérogations, de sanctions.

En Italie les premiers économistes ne furent pas des scientifiques, ce furent les praticiens, les marchands et les banquiers lombards. Ils ont laissé à l'Azienda Italia (l'entreprise Italie) un discours fondé sur la comptabilité commerciale générale, même si l'influence française l'a amenée à accueillir aussi un discours de type médical. 


\section{LA PROBLÉMATIQUE DE LA VARIABILITÉ DES MODÈLES DE DISCOURS POUR LE TRADUCTEUR}

Si l'on s'en tient à l'exemple choisi des discours économiques, on comprend aisément le problème qui se pose au traducteur. Si le traducteur français traduit un texte économique allemand en français en gardant le modèle de discours allemand, c'està-dire le modèle juridique, il produira un texte que les économistes français considéreront comme ne faisant pas partie de leur science. Car, il faut bien le dire, les praticiens des sciences économiques ont intériorisé à tel point leur modèle de discours étranger à leur science qu'ils n'admettent plus l'existence d'un tel modèle et qu'ils considèrent que c'est simplement le discours universel de l'économie. Pour le traducteur, la seule voie consiste donc à maîtriser suffisamment le modèle de discours des spécialistes de sa langue cible pour le faire entrer entièrement dans ses traductions quel que soit le modèle adopté par les auteurs de sa langue source.

\section{BIBLIOGRAPHIE}

A bdel H. M. (1992), Géographie politique et traduction juridique, le problème de la terminologie, Terminologie et traduction, $\mathrm{N}^{\circ} 2-3$, Luxembourg Commission des Communautés Européennes, pp. 43-56.

Altamore G. (1994), Tutte le parole dell'economia, Arnoldo Mondadori, Milano.

B ocquet C. (1994), Traduire les textes économiques financiers bancaires allemands aux différents niveaux du discours, C.B. Service S.A., Prilly-Lausanne.

- (1992), Phraséologie et traduction dans les langues de spécialités, Terminologie et traduction,

$\mathrm{N}^{\mathrm{o}} 2-3$, Commission des Communautés Européennes, pp. 271-284.

- (1988), Le droit comme signifiant, Parallèles, $N^{0}$ 9, ETI, Genève, pp. 59-63.

Cary E. (1985), Comment faut-il traduire, Presses universitaires de Lille, Lille.

- (1956), La traduction dans le monde moderne, publication de l'Ecole d'Interprètes de l'Université de Genève, Georg, Genève.

Cesari F., (1994), Alcune notazioni sulla traduzione economica, Parallèles, spécial symposium Banque et Finance, $N^{\circ} 16$, ETI, Genève, pp. 91-97 (GB-I).

Cipolla C. M. (1988), Tra due culture. Introduzione alla storia economica, Società editrice II Mulino, Bologna.

Colson J.-P., (1992), Ebauche d'une didactique des expressions idiomatiques en langues étrangères, Terminologie et traduction, $\mathrm{N}^{\circ} 2-3$, Luxembourg Commission des Communautés Européennes, pp. 165-180.

Dance tte J., (1992), La complexité de la langue économique et commerciale au Québec, les défis du traducteur, Terminologie et traduction, $\mathrm{N}^{\mathrm{o}} 2-3$, Luxembourg Commission des Communautés Européennes, pp. 197-210.

De Dax F. (1997), De la traduction générale à la traduction technique: une différence dans l'approche du lexique, in: L'histoire et les théories de la traduction, ASTTI Berne, et ETI Genève, pp. 505-514. 
Didaoui M., (1997-1998), Les conséquences politiques, juridiques et commerciales de l'erreur en traduction, Parallèles $\mathrm{N}^{\circ} 19$, Pistes de réflexion et de recherche en traduction, Genève, pp. 45-62.

Didier E., (1991), Le Common Law en français: Etude juridique et linguistique de la Common Law en français au Canada, Revue internationale de droit comparé, 1, pp. 7-56.

Dolet E. (1540), La manière de bien traduire d'une langue en aultre, à Lyon chez Dolet même.

Dullion V., (1997), Lorsque traduire, c'est écrire une page de l'histoire: la version française du Code Civil suisse dans l'unification juridique de la Confédération, in L'histoire et les théories de la traduction, ASTTI Berne et ETI Genève, pp. 371-388.

D u rie u x Ch., (1992), La terminologie en traduction technique: apports et limites, Terminologie et traduction, $\mathrm{N}^{\circ}$ 2-3, Commission des Communautés Européennes, pp. 95-104.

Fischer F., (1992), Le metafore in commercio, Italiano e oltre, $\mathrm{N}^{\circ} 7$, pp. 229-231.

Forsthoff E. (1971), Recht und Sprache. Prolegomena zu einer richterlichen Hermeneutik: Schriften der Königsberg Gelehrten-Gesellschaft, 17, Darmstadt.

Gall a is-Hamonno J. (1982), Langage, langue et discours économiques, Klincksieck, Paris.

Gémar J.-C., (1970), Réflexions sur le langage du droit: problèmes de langue et de style, META, vol. 26, pp. 338-349.

Grzegorczyk Ch. (1974), Le rôle du performatif dans le langage du droit, in: Archives de philosophie du droit, t. 19, Le langage du droit, Paris, pp. 229-241.

Ha ba E. P. (1974), Etude en allemand sur le rapport entre droit et langue, in: Archives de philosophie du droit, t. 19, Le langage du droit, 1974, Paris, pp. 257-290.

$11 \mathrm{~g}$ G. (1974), Le traducteur de langue française à la tâche, Symposium Banque et Finances, Parallèles $\mathrm{N}^{\circ} 16$, Genève, pp. 79-84.

Juglar C. (1860), Les crises commerciales et leur retour périodique en France, en Angleterre et aux Etats-Unis, Paris.

Lad mi ral J.-R. (1994), Traduire: théorèmes pour la traduction, réédition, Gallimard, Collection Tel, Paris.

- (1997), Les 4 âges de la traductologie - Réflexions sur une diachronie de la théorie de la traduction, in: L'histoire et les théories de la traduction, ASTTI Berne et ETI Genève, pp. 11-42.

Marazzi Ch. (1997), La place des chaussettes: le tournant linguistique de l'économie et ses conséquences politiques, trad. de l'italien par François Rosso et Anne Querrien, L'Eclat, Paris.

Mounin G. (1974), La linguistique comme science auxiliaire dans les disciples juridiques, in: Archives de philosophie du droit, t. 19, Le langage du droit, Sirey, Paris.

Pasqua relli G. (1987), Passaparola: parole nuove e neonuove, in: Economia, politica e costume, Edizione del Sole 24 Ore, Milano.

Pienkos J. (1990), La jurilinguistique et la traduction (problèmes théoriques et pratiques), in: Acta Univerzita Karleva, Prague, pp. 121-203.

Quesnay F. Oeuvres économiques et politiques éditées par A. Onken en 1888.

Recanati F. (1981), Les énoncés performatifs, Les Editions de Minuit, Paris.

Reiß K. (1971), Möglichkeiten und Grezen der Ubersetzungskritik, Hüber Hochschulerei 12, Max Huberverlag, Münich.

Roscher W. (1874), Grundlagen der Nationaloekonomie, Hannover.

Schmoller G. (1901), Grundriss der Allgemeinen Volkswirtschaftslehre, Berlin.

Sourioux J.-L., Lerat P. (1975), Le langage du droit, Presses universitaires de France, Coll. SVP, Le Juriste, Paris. 